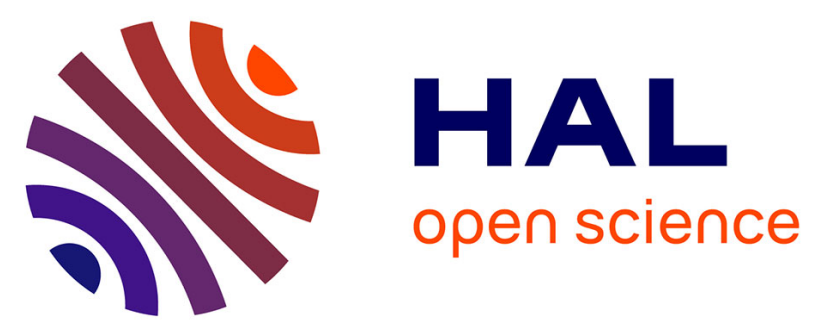

\title{
RNA-mediated interference and reverse transcription control the persistence of RNA viruses in the insect model Drosophila
}

Bertsy Goic, Nicolas Vodovar, Juan A Mondotte, Clément Monot, Lionel Frangeul, Hervé Blanc, Valérie Gausson, Jorge Vera-Otarola, Gaël Cristofari, Maria-Carla P Saleh

\section{To cite this version:}

Bertsy Goic, Nicolas Vodovar, Juan A Mondotte, Clément Monot, Lionel Frangeul, et al.. RNAmediated interference and reverse transcription control the persistence of RNA viruses in the insect model Drosophila. Nature Immunology, 2013, 14 (4), pp.396-403. 10.1038/ni.2542 . pasteur01957212

\section{HAL Id: pasteur-01957212}

\section{https://hal-pasteur.archives-ouvertes.fr/pasteur-01957212}

Submitted on 17 Dec 2018

HAL is a multi-disciplinary open access archive for the deposit and dissemination of scientific research documents, whether they are published or not. The documents may come from teaching and research institutions in France or abroad, or from public or private research centers.
L'archive ouverte pluridisciplinaire HAL, est destinée au dépôt et à la diffusion de documents scientifiques de niveau recherche, publiés ou non, émanant des établissements d'enseignement et de recherche français ou étrangers, des laboratoires publics ou privés.

\section{(ㅇ)(1)(2)}

Distributed under a Creative Commons Attribution - NonCommercial - ShareAlikel 4.0 
RNA interference and reverse transcription control RNA virus persistence in the insect model Drosophila

Bertsy Goic ${ }^{1}$, Nicolas Vodovar ${ }^{1,3}$, Juan A Mondotte ${ }^{1}$, Clément Monot ${ }^{2}$, Lionel Frangeul ${ }^{1}$, Hervé Blanc $^{1}$, Valérie Gausson ${ }^{1}$, Jorge Vera-Otarola ${ }^{2}$, Gael Cristofari ${ }^{2}$ \& Maria-Carla Saleh ${ }^{1}$

${ }^{1}$ Institut Pasteur, Viruses and RNA Interference, Centre National de la Recherche Scientifique URA3015, Paris, France. ${ }^{2}$ Institut National de la Santé et de la Recherche Médicale U1081, Centre National de la Recherche Scientifique Unité Mixte de Recherche 7284, University of NiceSophia-Antipolis, Faculty of Medicine, Institute for Research on Cancer and Aging, Nice, Nice, France. ${ }^{3}$ Present address: Institut National de la Santé et de la Recherche Médicale, Unité Mixte de Recherche en Santé 942, Hôpital Lariboisière, Paris, France; Département de Biologie, Institut de Biologie Génétique et Bioinformatique, Université d'Evry-Val-d'Essonne, Evry, France; Assistance Publique des Hôpitaux de Paris, Groupement Hospitalier Universitaire, Hôpital Lariboisière, Service de Biochimie et de Biologie moléculaire, Unité de Biologie Clinique Structurale, Paris, France. Correspondence should be addressed to M.-C.S.

(carla.saleh@pasteur.fr).

Received 9 November 2012; accepted 8 January 2013; published online XX Month 2013; doi:10.1038/ni.2542.

How persistent viral infections are established and maintained is widely debated and remains poorly understood. We found here that the persistence of RNA virus in Drosophila melanogaster was achieved through the combined action of cellular reverse-transcriptase activity and the RNA interference (RNAi) pathway. Fragments of diverse RNA viruses were reverse-transcribed early during infection, which resulted in DNA forms embedded in retrotransposon sequences. Those virus-retrotransposon DNA chimeras produced transcripts processed by the RNAi machinery, which in turn inhibited viral replication. Conversely, inhibition of reverse transcription hindered the appearance of chimeric DNA and prevented persistence. Our results identify a cooperative function for retrotransposons and antiviral RNAi in the control of lethal acute infection for the establishment of viral persistence.

The most well-characterized viral infections are those with human or economic effects. However, regardless of the organism under consideration, there are viruses able to infect that organism. Viral fossil registers highlight the long coevolutionary history between virus and 
host $^{1,2}$. The outcome of such host-pathogen interactions is highly variable and ranges from deleterious infections with lethal or permanent damage to completely innocuous infections ${ }^{3}$. For example, acute viral infections are characterized by a high rate of viral replication and the production of a large number of progeny. Replication is transient and is limited either by death of the infected cells or by clearance of the virus by the host immune response. In contrast, persistent infections may be the result of an acute primary infection that is not cleared. In this case, the ability of the virus to be transmitted to other organisms or to the offspring of the host is maintained. Persistent infections are at the boundary that separates deleterious infections from innocuous infections. In this unique circumstance, the virus and host use attack and counterattack machinery to reach an equilibrium at which viral infection is controlled but not eliminated. Insect-virus interactions are useful models with which to delineate persistent infections, because many insect viruses develop a persistent infection without clear fitness costs to the host ${ }^{4,5}$. Furthermore, many persistently infected arthropods, and insects in particular, can act as vectors for emerging viral infectious diseases with a strong medical and economic effect, such as West Nile Virus or Dengue virus ${ }^{6}$.

Flock house virus (FHV) belongs to the Nodaviridae family and is a nonenveloped virus with a bisegmented genome (RNA1, 3,107 nucleotides; RNA2, 1,400 nucleotides) of positive single-stranded RNA with a 5' terminal methylated cap and a nonpolyadenylated $3^{\prime}$ end. FHV is a useful viral model because it can produce acute and persistent infection in cell culture as well as in animal models $s^{7,8}$. Initial efforts to characterize persistent infections in vitro indicated that the FHV genome is unaltered during the establishment of persistence and that mutations on the viral genome begin to accumulate only after multiple passages on persistently infected cells ${ }^{9}$. Of note, mutations accumulate in RNA2, which encodes the coat protein, but not in RNA1, which encodes the viral RNA-dependent RNA polymerase and B2, a strong suppressor of RNA-mediated interference $(\mathrm{RNAi})^{10}$. Those observations suggest that a change in the cellular physiology rather than the virus itself is responsible for establishing the persistent state. However, the molecular and cellular mechanisms underlying this process have remained unsolved. Other studies have associated the appearance of defective interfering particles with persistent infection with $\mathrm{FHV}^{9,11}$ or other RNA viruses ${ }^{12-15}$. Defective interfering particles are unable to complete a full replication cycle because of genome deletions and consequently need wild-type viruses to replicate their genomes. Such particles can also interfere with the 
replication of wild-type virus through competition for viral or host factors essential for replication, facilitated by their replicative advantage due to the smaller size of their genome ${ }^{16}$. It has been suggested that during persistent infection of Drosophila melanogaster cells with FHV, the RNA derived from such particles is a chief contributor to the formation of virus-derived small interfering RNA (vsiRNA), because double-stranded RNA (dsRNA) from defective interfering particles could be processed more efficiently by the RNAi machinery than viral dsRNA replicative intermediates ${ }^{11,17}$. The RNAi machinery is also important in maintaining persistent infections in Drosophila cell lines ${ }^{18}$. That study suggests that direct dicing of the viral dsRNA replicative intermediate might be one mechanism that allows control of the viral infection, as the bulk of FHV-derived siRNAs are not loaded into the RNA-silencing effector proteins Argonaute 1 and Argonaute 2 (Ago2). Even if the 'dicing hypothesis' was able to explain how viral replication is controlled during long-lasting infections, it does not explain how the persistent state is established, mainly because that study used cells already persistently infected with FHV.

In this work, we sought to understand how viral persistence is established and maintained in insects. We found that Drosophila cells and flies infected with FHV or other positive single-stranded RNA viruses generated DNA of viral origin through endogenous reverse-transcriptase activity. We further demonstrated that those viral DNA forms were transcribed and produced vsiRNAs that 'fed' the RNAi antiviral machinery.

\section{RESULTS}

\section{Characterization of persistently infected Drosophila S2 cells}

To study how viral persistence is established and maintained in insects, we infected naive Drosophila S2 cells (S2n) by limiting dilution ${ }^{9,11}$ with several RNA viruses, including the positive single-stranded RNA viruses FHV and Drosophila C virus (DCV), and the dsRNA virus Drosophila $X$ virus (DXV; Supplementary Fig. 1a). Cells that survived the lytic infection proliferated and remained persistently infected even after 35 passages (Supplementary Fig. 1b). We further characterized the S2 cell lines persistently infected with FHV (S2p). Immunostaining of S2p cells with antibody to FHV capsid showed that all cells were homogeneously infected by FHV (Fig. 1a), which excluded the possibility of the presence of cells refractory to infection. Furthermore, S2p cells did not show a difference in proliferation (Fig. 1b) or death (Fig. 1C) relative to that of 
S2n cells, which indicated that persistent infection did not impose any 'fitness cost' on the S2p cell population. To exclude the possibility that selection of rare initial events contributed to the establishment of persistence independently of the virus, we tested the resistance of $S 2 p$ cells to apoptosis. Both S2p and S2n cells were similarly sensitive to ultraviolet irradiation (Fig. 1d) indicating that S2p survival to infection was not due to a defect in apoptosis. We also tested the infectivity of the virus produced by S2p cells. Wild-type $\left(w^{1118}\right)$ flies infected with $500 \mathrm{TCID}_{50}$ (half-maximal tissue culture infectious dose) of virus recovered from the supernatants of either S2p cells or acutely infected S2n cells died at a similar rate (Fig. 1e), which indicated that persistence was not established from a less-virulent virus population or from a loss of virulence during infection. As neither cell fitness nor FHV virulence was altered in S2p cells, we next hypothesized that the persistence could have resulted from the control of viral replication below a cytopathogenic threshold that would be accompanied by lower production of virus in S2p cells ${ }^{11}$. We compared viral titers after acute and persistent infection and observed that viral titers were significantly lower in S2p cells (Fig. 1f); accordingly, there was also less accumulation of viral RNA segments during persistent infection (Supplementary Fig. 1c). As viral titers varied in S2n cells versus S2p cells, we analyzed differences in the antiviral response. In insects, the main antiviral response acts through the canonical Dicer-2-Ago2 siRNA pathway ${ }^{10,19-21}$. To assess the antiviral RNAi response in S2p and acutely infected S2n cells, we produced small RNA libraries and examined the vsiRNA profiles. vsiRNAs that mapped all along both FHV RNA1 and RNA2 segments were found (Supplementary Fig. 1d-g), which indicated that the RNAi machinery effectively processed the viral dsRNA in both conditions. Together these observations showed that persistently infected cells produced less virus because of control of viral replication by an unknown cellular mechanism.

\section{New cellular synthesis of viral cDNA from viral RNA}

RNA from non-retroviral RNA viruses can be reverse-transcribed into cDNA by retrotransposons or endogenous retroviruses ${ }^{22-30}$. The role of non-retroviral DNA forms of RNA viruses remains unresolved, although involvement in immunity has been proposed ${ }^{25,26}$. Hence, we investigated whether RNA viruses generated DNA forms in Drosophila cells and whether those DNA forms correlated with the establishment and maintenance of persistent infection. We extracted genomic DNA from S2 cell lines persistently infected with FHV, DCV or DXV and amplified the DNA with primers located in various regions of the viral genomes. All samples contained DNA 
sequences (Fig. 2a and Supplementary Fig. 2a,b), a result we further confirmed by sequencing. Sindbis virus, an arbovirus that naturally produces persistent infection in insects, also produced a DNA form (Supplementary Fig. 2c). When we treated DNA samples with RNase III, a mixture of RNase A and RNAse I, DNase I or exonuclease I, only DNase I precluded the generation of a PCR product (Supplementary Fig. 2d and data not shown), which confirmed that the molecular template was a DNA molecule.

Through the use of 'genome walking', we extended the initially identified sequences, corresponding to the FHV RNA1. We reconstructed the FHV DNA forms present in S2p cell lines (Fig. 2a and Supplementary Fig. 3a). The DNA form was heavily reorganized, with a major recombinant RNA1 segment considerably shorter than the usual 3107 nucleotides (Supplementary Fig. 3a,b). Nonhomologous RNA recombination during negative-strand synthesis of FHV RNA1 and RNA2 could have been the template for those new DNA structures $^{31}$. Alternatively, defective interfering particles could have served as a template ${ }^{32,33}$, as the DNA forms had breakpoints and rearrangements similar to those identified in RNA1 defective interfering particles ${ }^{11}$. Of note, we also identified DNA forms derived from FHV RNA2 that were similar in sequence to RNA2 defective interfering particles ${ }^{32}$ (data not shown). We then infected S2n cells with FHV at a multiplicity of infection (MOI) of 0.5 and monitored the appearance of FHV DNA over time by PCR. The DNA form was detectable as early as $12 \mathrm{~h}$ after infection (Fig. 2b).

Because reverse transcriptases encoded by retrotransposons and endogenous retroviruses are widespread in insect genomes ${ }^{34,35}$, we determined if we could detect reversetranscriptase activity in S2 cells. We detected robust $\mathrm{Mn}^{2+}$-dependent reverse-transcriptase activity in extracts of S2n cells (Fig. 2c). Additionally, we found that such activity was sensitive in vitro to the nucleoside reverse-transcriptase inhibitor azidothymidine (AZT) triphosphate to a degree similar to that achieved by a recombinant retroviral reverse transcriptase (Fig. 2d). Next we determined whether treating S2n cells with AZT would inhibit the appearance of FHV DNA after infection with FHV. Indeed AZT triggered a dose-dependent inhibition of FHV DNA in S2n cells infected with FHV at an MOI of 0.5 (Fig. 2e). We confirmed that AZT did not impair S2 cell growth at the concentrations and time used (Supplementary Fig. 4a). Moreover AZT did not inhibit FHV replication in persistently infected cells in which the DNA form was already present 
(S2p cells; Supplementary Fig. 4b,c). Therefore, AZT seemed to be specifically blocking the generation of viral DNA rather than affecting cell or virus viability.

As mitochondrial dysfunction is a known potential side effect of AZT, and as FHV replicates on the mitochondrial external membrane, we also tested the effect of AZT treatment on other viruses, such as DCV and Sindbis virus, whose replication is not associated with mitochondria. In S2 cells, $5 \mathrm{mM}$ AZT also inhibited the synthesis of a viral DNA form after infection with DCV or Sindbis virus at a dose of $0.5 \mathrm{MOI}$ (Fig. 2f,g). Finally to rule out the possibility of any effects specific to S2 cells, we also analyzed the generation of FHV DNA forms and its inhibition by treatment with AZT in another Drosophila cell line, Kc167. The appearance of FHV DNA after infection of these cells (Fig. $\mathbf{2 h}$ ) indicated that our results were not unique to S2 cells but were instead a general characteristic of insect cells. Thus, during the establishment of viral persistence, RNA viruses and/or their defective interfering particles were reversetranscribed into viral DNA forms by host reverse transcriptase(s) in cultured Drosophila cells of various origins and AZT inhibited that process.

\section{The viral DNA form mediates persistent infection}

To determine whether the absence of a viral DNA form affects the antiviral response during the establishment of persistence, we deep-sequenced vsiRNA from FHV-infected S2n cells treated with AZT or not. At similar amounts of viral RNA (Supplementary Fig. 4d), the accumulation of vsiRNA was strongly impaired in cells treated with AZT and thus in the absence of FHV DNA forms (Fig. 3a and Supplementary Fig. 4e). In contrast, the global amount of miRNA remained unchanged despite AZT treatment (Fig. 3a), which indicated that at the doses and time used, AZT did not have pleiotropic effects. We then characterized the sequence diversity of vsiRNA reads. Cells showing the DNA form have vsiRNAs that mapped to the junctions of the DNA form rearrangements, whereas those vsiRNAs were undetectable in cells treated with AZT (Table 1). Those results suggested that the FHV DNA form was transcribed and processed into specific vsiRNAs. To determine whether inhibition of the synthesis of FHV DNA and the associated lower amount and diversity of vsiRNAS affected the ability of S2 cells to control FHV replication, we measured viral load after prolonged exposure to AZT. When the DNA form was inhibited, the viral load was up to 1,000-fold higher than that of infected cells in which the DNA form was present (Fig. 3b). That higher viral titer when the appearance of FHV DNA was prevented was accompanied by more cell death (Fig. 3c), which indicated that the DNA form was needed to 
establish persistence. Together these observations emphasized the requirement for the viral DNA form at early time points during acute infection to improve the antiviral response and to allow the establishment of persistence.

\section{Retrotransposons provide reverse transcriptase activity}

Having linked the appearance of FHV DNA forms to the establishment of persistent infection, we next defined the mechanism by which protection was conferred. We hypothesized that determining the structure and the genomic location of FHV DNA would suggest a mode of action. We thus analyzed the genome of S2p cells by deep sequencing. Analysis of chimeric paired-end reads showed that most viral DNA forms (nine of ten) were fused to fragments corresponding to long-terminal repeat (LTR) retrotransposons, mainly 297 , blood, diver, micropia and invader2 elements (Fig. 4a and Supplementary Table 1). That result suggests that FHV RNA was reverse-transcribed by the reverse-transcriptase activity of a broad set of retrotransposons actively transcribed in S2 cells (Supplementary Fig. 5a). In some paired-end reads, we were able to identify the exact crossover point between micropia and FHV DNA (Fig. $\mathbf{4 b}$ and Supplementary Fig. 5b,c). That junction was one nucleotide distant from the end of the LTR of micropia, which would suggest a possible 'forced copy-choice' recombination mechanism, as has been proposed for the recombination between retrotransposons and lymphocytic choriomeningitis virus in mice ${ }^{24}$. Because of the repetitive and polymorphic nature of the retrotransposon sequences, we were unable to unambiguously assign chromosomal positions to these FHV DNA forms. Alternatively, the DNA-repair machinery can also process nuclear retroviral DNA to produce stable extrachromosomal circular molecules with a single LTR or two LTRs ${ }^{36}$; thus, we cannot exclude the possibility that the viral DNA form was located on such extrachromosomal molecules. In summary, these results indicated that LTR retrotransposons were the likely source of the reverse-transcriptase activity that produced FHV DNA fragments that were embedded in LTR retrotransposon DNA.

\section{Production of vsiRNAs from newly synthesized viral cDNA}

The presence of chimeric DNA molecules consisting of viral cDNA and retrotransposon DNA is not sufficient by itself to explain the mechanism by which persistence is reached. We thus hypothesized that a transcript from the FHV-retrotransposon DNA chimera might produce small RNAs that mediate protection against acute infection through the RNAi machinery, as 
suggested by the greater number and diversity of vsiRNAs observed (Fig. 3a,b). To assess the involvement of RNAi in this process, we depleted S2p cells of Dicer-2 (a core component of RNAi) ${ }^{18}$ or CG4572 (an uptake-spread component of RNAi) ${ }^{37}$ by knockdown via RNAi and measured cell death. The equilibrium between viral replication and persistence was broken in cells in which those genes were silenced by RNAi, which shifted the persistent infection to an acute infection that induced cell death (Fig. 4c). To further confirm the involvement of the RNAi response, we generated small RNA libraries from S2p cell lines. As each vsiRNA could originate from either replicating viral dsRNA (profiles, Supplementary Fig. 1d-g) or virus-retrotransposon chimeric transcripts, the only way to discriminate small RNAs specifically from the transcription of the DNA form was to identify those small RNAs whose sequence mapped partly to the virus and partly to the Drosophila genome. We expected these chimeric virus-Drosophila small RNAs to be very infrequent. To improve detection, we treated the samples to $\beta$-elimination (which prevents ligation on the 3 ' end of the RNAs unless they bear a 3 ' modification) to discriminate small RNAs loaded in Ago2 complexes and bearing a 3' 2'-O-methyl from the total small RNA background. The frequency at which such virus-Drosophila small RNA chimeras occurred ranged from 1.15 to 2.3 per 10,000 total unique sequences (Fig. 4d). Indeed, in S2p cells, we identified over 899 chimeric small-RNA reads unambiguously identified and loaded in Ago2 (241 and 427 for S2p 1 cell lines a and b, respectively, and 231 for the S2p 2 cell line) when we aligned smallRNA libraries with the FHV and the Drosophila genome reference sequence. We further confirmed the existence of those chimeric small RNAs by analyzing publicly available small-RNA libraries generated from persistently infected S2 cells in other laboratories (Supplementary Fig. 6). Of note, in the libraries analyzed, all the chimeric reads mapped to retrotransposons on their Drosophila part, and $\sim 65 \%$ of their virus-derived sequences matched the positive strand of FHV. Thus, the presence of chimeric small RNAs mapping partly to Drosophila retrotransposons and partly to FHV further confirmed that the RNA was transcribed from FHV DNA templates and was processed by the siRNA machinery into vsiRNA.

\section{Inhibiting virus DNA form increases viral load in vivo}

To determine if mechanisms similar to those described above could be involved in viral persistence in vivo, we infected wild-type flies with $20 \mathrm{TCID}_{50} \mathrm{FHV}$ and monitored the appearance of the FHV DNA form over time by PCR of single flies. We detected fragments of FHV DNA in vivo from day 4 onward (Fig. 5a). The characterization of the FHV RNA1 sequence of 
these DNA forms identified an almost complete full-length DNA as well as reorganized forms similar to those present in S2p cells (Supplementary Fig. 3c). The appearance of a DNA form in infected flies was a common event, with $84.7 \%$ of 200 FHV-infected flies having a DNA form and $58 \%$ of 200 Sindbis virus-infected flies having a DNA form at day 6 after injection.

To assess the effect of the DNA form on the antiviral response in vivo, we developed a protocol for natural inoculation with FHV by feeding. We maintained flies in the presence of $25 \%$ sucrose and $93 \mathrm{mM} \mathrm{AZT} \mathrm{from} \mathrm{day} 2$ after eclosion. At day 4 after eclosion, we fed the flies overnight pure FHV stock $\left(1 \times 10^{9} \mathrm{TCID}_{50}\right.$ per $\left.\mathrm{ml}\right)$, then monitored survival every day for $16 \mathrm{~d}$. After that natural infection protocol, we found that flies infected with FHV but not treated with AZT controlled viral infection (Fig. 5b) and had a death rate undistinguishable from uninfected flies (Fig. 5c). In contrast, when treated with AZT, infected flies were unable to contain virus replication as shown by the high viral titers at day 13 (Fig. 5b), and $>75 \%$ of the flies died within $13 \mathrm{~d}$ of infection (Fig. 5c). In control experiments, uninfected flies treated with AZT had a low death rate over the course of the experiment, which excluded the possibility of considerable pleiotropic effects of AZT alone (Fig. 5c). Of note, we originally developed a double-injection protocol in which flies were injected daily intrathoracically with AZT and challenged them with FHV. This protocol proved to be lethal for the flies above 4 days because of repeated physical injury; however, when analyzed, this injection protocol yielded a similar result: in the absence of a DNA form, infected flies died because of an increase in viral replication (data not shown). Collectively, these results confirmed that inhibition of FHV DNA synthesis affected the establishment of persistent infection and demonstrated a role for the DNA form in antiviral immunity in vivo. Together our data demonstrated that RNAi and retrotransposons acted together to establish and maintain viral persistent infection in insects; these results provide a mechanistic framework for understanding this process (Fig. 6).

\section{DISCUSSION}

The host-pathogen interaction triggers selection pressures on both organisms that drive the development of survival strategies. This survival sometimes indicates the incorporation or endogenization of the full parasitic organism by the host, as noted for the endosymbiont bacterium Wolbachia, which protects fruit flies and mosquitoes against infection with several viruses $^{38,39}$. In other cases, only part of the parasitic genome is endogenized ${ }^{26}$; for example, bees whose genomes have integrated fragments of Israeli acute paralysis virus are resistant to 
further challenge with that virus ${ }^{26}$. Our results have demonstrated that one possible root of viral persistence, commonly observed in insects and other arthropods ${ }^{40}$, is the endogenization of viral RNA sequences. Indeed, the establishment of persistent viral infection depends on the formation of viral cDNA fragments from which small RNAs are produced by the RNAi machinery. We postulate that viral dsRNA, the canonical substrate of the antiviral RNAi machinery, is also generated from viral cDNA. The biogenesis of that dsRNA remains unknown and should be the subject of future research. However, we speculate that dsRNA might originate from a singlestranded viral transcript generated from the DNA form annealed to the viral genome (either the positive or negative strand, depending on the orientation of the transcript). Another possibility is that a single-stranded viral transcript generated from the DNA form folds back on itself and forms double-stranded secondary structures that could be recognized by Dicer and enters into the RNAi pathway, similar to endogenous siRNA. A third possibility is two complementary single-stranded viral transcripts generated from different loci or by convergent transcription. When the DNA form is inhibited, dsRNA produced through one or several of these mechanisms ${ }^{3}$ disappears, with a consequent decrease in vsiRNA.

Given our data, we propose the following model to explain the establishment and maintenance of persistent infection with RNA viruses in insects. After viral infection, ongoing viral replication is limited either by the death of the infected cell or by the antiviral RNAi response in the host. During that process, viral RNA is reverse-transcribed by endogenous reverse-transcriptase activity of LTR retrotransposons. The resulting DNA molecule can then be imported in the nucleus, where retrotransposon-mediated integration into the host genome takes place ${ }^{34}$. Alternatively, the DNA-repair machinery can produce stable extrachromosomal circular DNA molecules that are efficiently transcribed ${ }^{36}$. In all cases, the viral DNA is continuously transcribed and produces dsRNA, which is recognized and processed by the RNAi machinery that boosts the antiviral response. It is possible that such dsRNA molecules are more exposed to Dicer- 2 than are viral replication intermediates, and then the resulting small RNAs are loaded into the RNA-induced silencing complex. When a small RNA that is transcribed and processed from a viral DNA form reaches that complex, the ongoing infection can be better contained and controlled, as the immune response is already primed. In this way, both cell and virus have time to reach a metastable equilibrium, or persistent infection. 
In the model propose, the interactions between two parasites (transposon and virus) and the RNAi pathways that control them determine the outcome of the infection. In our model, the basal protection afforded by RNAi during viral infection and the priming of the RNAi response in uninfected cells ${ }^{37}$ are key to providing the time the cell needs to initiate the persistence mechanism and to control viral infection. In this way, the virus-transposon interaction serves an important role in the modulation of the immune system: the characteristically massive virus production followed by cell death in acute infection is compromised, yet viral dissemination in the persistent state is still ensured. We also speculate that in the absence of the canonical production of secondary small RNAs by RNA-dependent RNA polymerase in insects, this mechanism of transformation of viral RNA into DNA, then into RNA and finally into small RNA could be amplifying and maintaining the antiviral immune response throughout the insect's life after primary exposure. By the mechanism proposed, the RNAi immune response is triggered by viral dsRNA replication intermediates and is amplified and boosted through newly generated viral cDNA-derived dsRNA molecules. As defective interfering particles could be the template for new viral DNA synthesis, a similarity to interferon activation in mammalian cells can be seen. Indeed, viruses such as paramyxoviruses can activate the interferon cascade independently of viral protein synthesis but by a mechanism dependent on defective interfering particles ${ }^{41}$. In this model, the integrity of the defective interfering particle genomic RNA seems to be required for efficient interferon induction. In insects, the considerable sequence similarity among FHV DNA forms and defective interfering particles in different S2p cell lines in vitro, as well as in vivo, suggests a link between defective interfering particles and the biogenesis of viral DNA. Our results are compatible with two possibilities. In one, defective interfering particle RNAs are used as a template by retrotransposon reverse transcriptases to generate viral DNA forms. In the other, viral DNA is the template for the production of defective interfering particles. Further studies addressing this aspect could connect defective interfering particles to persistent infections and explain how these are linked.

Until now, endogenization of DNA has been considered a rare event, as it has been assumed that only endogenization in the germ line has an effect on host evolution. However, somatic (or 'nontransmissible') endogenization may be much more frequent than expected, as the restrictions on genome integrity in the soma could be more relaxed. DNA forms of 
nonretroviral viruses have been described in a wide variety of eukaryotic organisms, from

plants to mammals ${ }^{24,25,27-30}$. Whether those DNA forms are also involved in immunity mediated by small RNA or other types of immune responses in other organisms is an open question that deserves further exploration. The model proposed here offers a new perspective on antiviral immunity that considers persistent infection the result of the concerted effort of the host's multiple defense pathways.

\section{METHODS}

Methods and any associated references are available in the online version of the paper.

Accession codes. NCBI Small Read Archive: sequences, SRA045427.

Note: Supplementary information is available in the online version of the paper.

\section{ACKNOWLEDGMENTS}

We thank members of the Saleh lab, the Antoniewski lab and the Vignuzzi lab for discussions and technical support; M. Vignuzzi, J. Chandler and R. van Rij for critical reading of the manuscript; A. Schneemann (The Scripps Research Institute, La Jolla, CA) for antibody to FHV; P. Vargas for assistance with flow cytometry; and A. Pelisson for advice on retrotransposition. Supported by the French Agence Nationale de la Recherche (ANR-09-JCJC-0045-01 to M.-C.S.), the European Research Council (FP7/2007-2013 ERC 242703 to M.-C.S.), Institut National de la Santé et de la Recherche Médicale (for work in the laboratory of G.C.) the European Research Council (ERC 243312, Retrogenomics; for work in the laboratory of G.C.), the French Ministry of Research (C.M.), La Fondation ARC pour la Recherche sur le Cancer (C.M.) and the AXA Research Fund (J.A.M.).

\section{AUTHOR CONTRIBUTIONS}

B.G. and M.-C.S. designed the experiments, discussed the interpretation of the results and wrote the manuscript; G.C. participated in interpreting the data and writing the manuscript; B.G., N.V., J.A.M. and V.G. did research; H.B. generated the genomic DNA and the small-RNA libraries; N.V. and L.F. contributed to the bioinformatics analysis; and C.M., J.V.-O. and G.C. did the in vitro analysis of the reverse-transcriptase activity and AZT inhibition.

\section{COMPETING FINANCIAL INTERESTS}

The authors declare no competing financial interests. 
Reprints and permissions information is available online at

http://www.nature.com/reprints/index.html.

<jrn>1. Emerman, M. \& Malik, H.S. Paleovirology-modern consequences of ancient viruses. PLoS Biol. 8, e1000301 (2010).</jrn>

$<j r n>2$. Goldstone, D.C. et al. Structural and functional analysis of prehistoric lentiviruses uncovers an ancient molecular interface. Cell Host Microbe 8, 248-259 (2010).</jrn>

<jrn>3. Goic, B. \& Saleh, M.C. Living with the enemy: viral persistent infections from a friendly viewpoint. Curr. Opin. Microbiol. 15, 531-537 (2012).</jrn>

$<j r n>4$. Flegel, T.W. Update on viral accommodation, a model for host-viral interaction in shrimp and other arthropods. Dev. Comp. Immunol. 31, 217-231 (2007).</jrn>

$<$ jrn>5. Roekring, S., Flegel, T.W., Malasit, P. \& Kittayapong, P. Challenging successive mosquito generations with a densonucleosis virus yields progressive survival improvement but persistent, innocuous infections. Dev. Comp. Immunol. 30, 878-892 (2006).</jrn>

$<$ jrn>6. Weaver, S.C. \& Reisen, W.K. Present and future arboviral threats. Antiviral Res. 85, $328-345(2010) \cdot</ j r n>$

$<j r n>7$. Rechavi, O., Minevich, G. \& Hobert, O. Transgenerational inheritance of an acquired small RNA-based antiviral response in C. elegans. Cell 147, 1248-1256 (2011).</jrn>

$<j r n>8$. Dasgupta, R. et al. Replication of flock house virus in three genera of medically important insects. J. Med. Entomol. 44, 102-110 (2007).</jrn>

<jrn>9. Dasgupta, R., Selling, B. \& Rueckert, R. Flock house virus: a simple model for studying persistent infection in cultured Drosophila cells. Arch. Virol. Suppl. 9, 121-132 $(1994) .</ j r n>$

<jrn>10. Li, H., Li, W.X. \& Ding, S.W. Induction and suppression of RNA silencing by an animal virus. Science 296, 1319-1321 (2002).</jrn>

$<j r n>11$. Jovel, J. \& Schneemann, A. Molecular characterization of Drosophila cells persistently infected with Flock House virus. Virology 419, 43-53 (2011).</jrn> 
$<$ jrn $>12$ Ebner, P.D., Kim, S.K. \& O'Callaghan, D.J. Biological and genotypic properties of defective interfering particles of equine herpesvirus 1 that mediate persistent infection. Virology 381, 98-105 (2008).</jrn>

$<$ jrn>13. Tsai, K.N., Tsang, S.F., Huang, C.H. \& Chang, R.Y. Defective interfering RNAs of Japanese encephalitis virus found in mosquito cells and correlation with persistent infection. Virus Res. 124, 139-150 (2007).</jrn>

$<j r n>14$. Simon, A.E., Roossinck, M.J. \& Havelda, Z. Plant virus satellite and defective interfering RNAs: new paradigms for a new century. Annu. Rev. Phytopathol. 42, 415$437(2004) .</ j r n>$

$<j r n>15$. Huang, A.S. \& Baltimore, D. Defective viral particles and viral disease processes. Nature 226, 325-327 (1970).</jrn>

$<$ jrn>16. Marriott, A.C. \& Dimmock, N.J. Defective interfering viruses and their potential as antiviral agents. Rev. Med. Virol. 20, 51-62 (2010).</jrn>

<jrn>17. Vodovar, N., Goic, B., Blanc, H. \& Saleh, M.C. In silico reconstruction of viral genomes from small RNAs improves virus-derived small interfering RNA profiling. J. Virol. 85, 11016-11021 (2011).</jrn>

<jrn>18. Flynt, A., Liu, N., Martin, R. \& Lai, E.C. Dicing of viral replication intermediates during silencing of latent Drosophila viruses. Proc. Natl. Acad. Sci. USA 106, 5270-5275 (2009). $</$ jrn $>$

<jrn>19. Galiana-Arnoux, D., Dostert, C., Schneemann, A., Hoffmann, J.A. \& Imler, J.L. Essential function in vivo for Dicer-2 in host defense against RNA viruses in Drosophila. Nat. Immunol. 7, 590-597 (2006).</jrn>

<jrn>20. van Rij, R.P. et al. The RNA silencing endonuclease Argonaute 2 mediates specific antiviral immunity in Drosophila melanogaster. Genes Dev. 20, 2985-2995 (2006).</jrn>

$<$ jrn>21. Wang, X.H. et al. RNA interference directs innate immunity against viruses in adult Drosophila. Science 312, 452-454 (2006).</jrn> 
<jrn>22. Belyi, V.A., Levine, A.J. \& Skalka, A.M. Unexpected inheritance: multiple integrations of ancient bornavirus and ebolavirus/marburgvirus sequences in vertebrate genomes. PLoS Pathog. 6, e1001030 (2010).</jrn>

$<j r n>23$. Crochu, S. et al. Sequences of flavivirus-related RNA viruses persist in DNA form integrated in the genome of Aedes spp. mosquitoes. J. Gen. Virol. 85, 1971-1980 $(2004) .</ j r n>$

$<j r n>24$. Geuking, M.B. et al. Recombination of retrotransposon and exogenous RNA virus results in nonretroviral cDNA integration. Science 323, 393-396 (2009).</jrn>

<jrn>25. Klenerman, P., Hengartner, H. \& Zinkernagel, R.M. A non-retroviral RNA virus persists in DNA form. Nature 390, 298-301 (1997).</jrn>

$<$ jrn>26. Maori, E., Tanne, E. \& Sela, I. Reciprocal sequence exchange between non-retro viruses and hosts leading to the appearance of new host phenotypes. Virology 362, 342-349 (2007).</jrn>

$<j r n>27 . \quad$ Chiba, S. et al. Widespread endogenization of genome sequences of nonretroviral RNA viruses into plant genomes. PLoS Pathog. 7, e1002146 (2011).</jrn> <jrn>28. Horie, M. et al. Endogenous non-retroviral RNA virus elements in mammalian genomes. Nature 463, 84-87 (2010).</jrn>

<jrn>29. Katzourakis, A. \& Gifford, R.J. Endogenous viral elements in animal genomes. PLoS Genet. 6, e1001191 (2011).</jrn>

$<j r n>30$. Liu, H. et al. Widespread horizontal gene transfer from double-stranded RNA viruses to eukaryotic nuclear genomes. J. Virol. 84, 11876-11887 (2010).</jrn>

$<j r n>31$ Li, Y. \& Ball, L.A. Nonhomologous RNA recombination during negative-strand synthesis of flock house virus RNA. J. Virol. 67, 3854-3860 (1993).</jrn>

<jrn>32. Zhong, W., Dasgupta, R. \& Rueckert, R. Evidence that the packaging signal for nodaviral RNA2 is a bulged stem-loop. Proc. Natl. Acad. Sci. USA 89, 11146-11150 $(1992) \cdot</ j r n>$

<jrn>33. Wu, Q. et al. Virus discovery by deep sequencing and assembly of virus-derived small silencing RNAs. Proc. Natl. Acad. Sci. USA 107, 1606-1611 (2010).</jrn> 
$<j r n>34$ Eickbush, T.H. \& Jamburuthugoda, V.K. The diversity of retrotransposons and the properties of their reverse transcriptases. Virus Res. 134, 221-234 (2008).</jrn>

<jrn>35. Terzian, C., Pelisson, A. \& Bucheton, A. Evolution and phylogeny of insect endogenous retroviruses. BMC Evol. Biol. 1, 3 (2001).</jrn>

$<$ jrn>36. Wu, Y. \& Marsh, J.W. Selective transcription and modulation of resting T cell activity by preintegrated HIV DNA. Science 293, 1503-1506 (2001).</jrn>

<jrn>37. Saleh, M.C. et al. Antiviral immunity in Drosophila requires systemic RNA interference spread. Nature 458, 346-350 (2009).</jrn>

<jrn>38. Teixeira, L., Ferreira, A. \& Ashburner, M. The bacterial symbiont Wolbachia induces resistance to RNA viral infections in Drosophila melanogaster. PLoS Biol. 6, e2 (2008). $</$ jrn $>$

<jrn>39. Bian, G., Xu, Y., Lu, P., Xie, Y. \& Xi, Z. The endosymbiotic bacterium Wolbachia induces resistance to dengue virus in Aedes aegypti. PLoS Pathog. 6, e1000833 (2010).</jrn>

$<$ jrn>40. Flegel, T.W. Hypothesis for heritable, anti-viral immunity in crustaceans and insects. Biol. Direct 4, 32 (2009).</jrn>

<jrn>41. Killip, M.J., Young, D.F., Precious, B.L., Goodbourn, S. \& Randall, R.E. Activation of the $\beta$ interferon promoter by paramyxoviruses in the absence of virus protein synthesis. J. Gen. Virol. 93, 299-307 (2012).</jrn>

Figure 1 Characterization of persistently infected Drosophila S2 cells and their viral population. (a) Immunostaining of persistently infected (S2p) and naive (S2n) S2 cells with polyclonal antibody to FHV (red), and with the DNA-intercalating dye DAPI (blue). Scale bars, $25 \mu \mathrm{m}$. (b) Cell-proliferation index of S2n (white) and S2p (gray) cells, quantified by staining with CFDA (carboxfluorescein diacetate succinymidyl ester) and flow cytometry, presented as the ratio of proliferation at $0 \mathrm{~h}$ to that at $48 \mathrm{~h}$. (c) Quantification of death in S2n and S2p cells stained with propidium iodide and assessed by flow cytometry 5 min later. (d) Viability of S2n and S2p cells (three independent groups of $2 \times 10^{6}$ cells each) $72 \mathrm{~h}$ after treatment with ultraviolet (UV) irradiation (increasing doses, horizontal axis). (e) Survival of wild-type flies $\left(w^{1118}\right)$ after intrathoracic injection of FHV produced during acute or persistent infection or after mock 
injection, monitored daily. 40 flies per group were injected and each condition was represented at least three times. (f) Viral titers in supernatants of S2p and S2n cells acutely infected with FHV at an MOI of 1 , quantified by endpoint dilution at $0 \mathrm{~h}$ (input) and $72 \mathrm{~h}$ after infection (below plot). Solid horizontal lines, median and s.d.; dotted lines, data dispersion (minimum to maximum). NS, not significant; ${ }^{*} P<0.02$ and ${ }^{*} * P<0.0001$ (Student's $t$-test). Data from one representative experiment out of four (a), three independent experiments (b-d; mean and s.d.), one representative experiment out of three (e; error bars, s.d.) or at least eight independent experiments (f).

Figure 2 Biogenesis of viral DNA forms during infection. (a) Structure of the main FHV RNA1 DNA form present in one $\$ 2 p$ cell line. Numbers indicate breakpoint coordinates. $n t$, nucleotide. (b) Kinetics of the appearance of FHV DNA forms in S2n cells left uninfected (UI) or infected with FHV (MOI, 0.5), followed by extraction of total DNA and RNA at 0-48 $\mathrm{h}$ after infection (above lanes) and treatment of DNA with DNase I (DNase I +) or not (DNase I-), then analysis by PCR and RT-PCR, respectively, with primers designed to amplify an FHV RNA1 sequence. Sequence encoding the ribosomal protein Rp49 serves as a control. (c) Endogenous reversetranscriptase activity in S2n cell extracts, assessed in the presence of $\mathrm{Mg}^{2+}$ or $\mathrm{Mn}^{2+}$ and various combinations of oligo(dT) and poly(rA), spotted onto DE81 paper (which retains poly(dT) products but not free $\left[{ }^{32} \mathrm{P}\right] \mathrm{dTTP}$ ) and measured at initial velocity phase. SSII, Superscript II used in place of S2n cell extract; Heat, heat-inactivated cells. Below, quantification of the spots above, in arbitrary units (AU). (d) Endogenous reverse transcriptase activity of in S2n cell extracts $(3.2 \mu \mathrm{M})$ and Superscript II $(1.8 \mu \mathrm{M})$ treated with AZT-triphosphate in vitro, presented as the half-maximal inhibitory concentration $\left(I_{50}\right)$ determined by nonlinear regression. (e) Appearance of FHV DNA form in S2n cells infected with FHV (MOI, 0.5$)$ in the presence $(1,5$ or $10 \mathrm{nM}$ ) or absence $(0 \mathrm{nM})$ of $\mathrm{AZT}$, then treated as in (b) at $24 \mathrm{~h}$ after infection. (f-h) Kinetics of the appearance of the DCV (f), Sindbis virus (g) or FHV (h) DNA form in S2 cells (f,g) or Kc167 cells (h) infected with DCV (f), Sindbis virus (g) or FHV (h) and treated as in b. Representative data from eight experiments (b), three experiments (c, e), two experiments (f-h). Data from three experiments (d; mean and s.e.m.).

Figure 3 The FHV DNA form improves the antiviral response via the RNAi machinery. (a) Accumulation of vsiRNA and cellular miRNA in S2 cells infected for $12 \mathrm{~h}$ with FHV in the presence $(\mathrm{AZT}(+))$ or absence $(\mathrm{AZT}(-))$ of $\mathrm{AZT}$, assessed as mapping by small RNA corresponding 
to each individual miRNA (blue) or to each FHV nucleotide for vsiRNA (red). (b) Viral titers in S2 cells given no pretreatment (0) or pretreated (5) with $5 \mathrm{mM} \mathrm{AZT} \mathrm{(AZT} \mathrm{before} \mathrm{inf),} \mathrm{then} \mathrm{infected}$ with serial dilutions of FHV with (5) or without (0) continued AZT treatment (AZT during inf), determined by end-point dilution (presented as in Fig. 1f). Presence (+) or absence (-) of the DNA form was monitored by PCR. (c) Survival of S2 cells after infection with FHV in the presence or absence of AZT, measured by exclusion of trypan blue dye. ${ }^{*} P<0.0001$ (Student's $t$-test). Data from one representative experiment out of two (a), at least eight independent experiments (b) or three independent experiments with three groups of 500 cells each per condition (c; mean and s.d.).

Figure 4 Viral-retrotransposon DNA chimeras produce transcripts processed by the RNAi machinery. (a) Paired-end genomic DNA deep sequencing of S2p cells, presented as reads of Drosophila DNA-FHV DNA form chimeras (numbers in plots). (b) Paired-end genomic DNA 'read' with a defined crossover point between the micropia LTR retrotransposon DNA and FHV cDNA. Numbers indicate nucleotide coordinates (distance in kilobases (kb) in key); long gray arrows, aligned 'read' pair; gray font, mismatched nucleotides. Gag-Pol, sequence encoding group-specific antigen and polymerase; PBS, primer-binding site; PPT, polypurine tract. (c) Death of S2n and S2p cells treated with scrambled sequence or RNAi directed the gene encoding Dicer-2 (Dcr-2) or CG4572, assessed by staining with propidium iodide and flow cytometry; results are presented relative to those in cells treated with RNAi directed at the gene Gal80 as a nonspecific control. (d) Frequency of small RNA in S2n cells, S2p cells (two independent lines, S2p 1 and S2p 2) and a technical replicate (S2p 1a and S2p 1b) in the presence $(+)$ or absence $(-)$ of $\beta$-elimination, assessed by deep sequencing of RNA libraries for the presence of chimeric sequences mapping partly to the virus and partly to Drosophila and presented as chimeras per $1 \times 10^{6}$ total unique sequences (only unique sequences representing at least five reads were included in the analysis). Data from one representative experiment (a), or three independent experiments (c).

Figure 5 The FHV DNA form is involved in viral persistence in vivo. (a) Kinetics of the formation of FHV DNA in single wild-type flies injected with Tris- $\mathrm{HCl}$ (-; control) or infected with FHV (20 $\left.\mathrm{TCID}_{50} ;+\right)$, followed by extraction of DNA and RNA, treatment DNase I and then PCR and RT-PCR (all as in Fig. 2b). (b) Viral titers in flies infected with FHV and treated with AZT (AZT(+)) or not $(A Z T(-))$, then homogenized in PBS at 0, 3 and $13 \mathrm{~d}$ after infection, determined by end-point 
dilution (presented as in Fig. 1f). ${ }^{* *} p<0.0001$ (Student's $t$-test). (c) Survival of wild-type flies $\left(w^{1118}\right)$ fed 93 mM AZT in sucrose daily $($ AZT $(+))$ or not (AZT(-)) and fed Tris-HCl (Mock) or FHV viral stock (FHV) once, monitored daily for $16 \mathrm{~d}$ after FHV feeding (horizontal axis). 30 flies per group were treated and each condition was repeated at least three times. Representative data from one experiment out of four (a), at least eight independent experiments with three pools of five flies per pool in each (b) or one representative experiment out of three (c; error bars, s.d.).

Figure 6 Model for the establishment and maintenance of viral persistent infection in insects. After viral infection, viral genomes (viral RNA) or dsRNA intermediates (viral dsRNA) are propagated (red). Those viral forms are reverse-transcribed by cellular reverse-transcriptase activity into DNA forms (green) that may integrate into the host genome or be processed into extrachromosomal circular DNA. The sequences of viral origin, now in a DNA form, will produce transcripts (black) that form dsRNA, recognized by Dicer 2 (Dcr2) and further processed by a small RNA-related pathway. When a viral small RNA from those transcripts reaches the RNAinduced silencing complex (RISC), the ongoing infection is contained and the acute infection is controlled. In this way, both cell and virus progress into a metastable equilibrium that defines the state of persistent infection.

Table 1: Abundance of vsiRNA covering FHV-FHV junctions in the presence or absence of AZT.

\begin{tabular}{|c|c|c|}
\hline \multicolumn{3}{|c|}{ \# of FHV-siRNA reads } \\
\hline Junction & AZT (-) & AZT (+) \\
\hline $311-957$ & 5 & 0 \\
\hline $301-967$ & 18 & 0 \\
\hline $318-944$ & 55 & 0 \\
\hline $1263-2275$ & 7 & 0 \\
\hline $1309-1941$ & 10 & 0 \\
\hline $2040-2189$ & 78 & 0 \\
\hline
\end{tabular}




\section{ONLINE METHODS}

Cells and cell assays. Drosophila S2 cells (Invitrogen) and Kc167 cells were cultured at $25{ }^{\circ} \mathrm{C}$ in Schneider's Drosophila medium supplemented with 10\% heat-inactivated FCS. For cellproliferation assays, S2 cells were incubated for $15 \mathrm{~min}$ at room temperature in the presence of $2 \mu \mathrm{M}$ CFDA (5- (and 6-) carboxyfluorescein diacetate; Invitrogen) and were washed twice with PBS. For cell-viability assays, $1 \mu \mathrm{g} / \mathrm{ml}$ of propidium iodide was added to the cells, followed by incubation for $5 \mathrm{~min}$ at room temperature. Cellular fluorescence of $5 \times 10^{4}$ cells was analyzed at various times after staining with a FACSCalibur and CellQuest software. Alternatively (for Fig. 3d), for quantification of the viability of FHV-infected S2 cells in presence or absence of AZT, a portion of the infected cells was removed and stained for 5 min with $0.2 \%$ trypan blue (SigmaAldrich). For ultraviolet irradiation-induced DNA damage, cells were exposed to increasing doses of ultraviolet irradiation. At $72 \mathrm{~h}$ after irradiation, cell viability was measured by CellTiterGlo Luminescent Cell Viability Assay (Promega).

Viruses. FHV, DCV and DXV viral stocks were prepared on low-passage S2 cells and titers were measured by end-point dilution. S2 cells $\left(25 \times 10^{4}\right.$ cells per well in a 96 -well plates) were inoculated with tenfold dilutions of virus stocks. At $14 \mathrm{~d}$ after infection, cytopathic effects were analyzed. Viral titers were calculated as $\mathrm{TCID}_{50}$ (median tissue culture infective dose) according to a published method ${ }^{42}$.

For quantification of viral titers in flies, flies homogenized at various time in $250 \mu \mathrm{LBB}$, and titers in the homogenate were calculated as described above.

Sindbis viral stock was prepared in BHK hamster kidney cells and titers were measured by plaque assay.

Fly infection. For infection of flies by injection, $w^{1118}$ flies were used as wild-type controls; these were reared on standard medium at $25{ }^{\circ} \mathrm{C}$. Four-day-old female flies were injected intrathoracically with $50 \mathrm{nl}$ of a FHV dilution in $10 \mathrm{mM}$ Tris- $\mathrm{HCl}\left(\mathrm{pH} \mathrm{7.5)}\right.$ as described ${ }^{43}$, with a Nanoject II injector. For analysis of survival, FHV was injected at a dose of 500 TCID $_{50}$ per fly. Mock-infected flies were injected with $10 \mathrm{mM}$ Tris- $\mathrm{HCl}, \mathrm{pH}$ 7.5. Fly mortality at day 1 was 
attributed to damage produced by the injection procedure and those data were excluded from further analysis. Mortality was monitored daily for $14 d$, and every 3-4 d the flies were transferred to fresh food.

For in vivo viral DNA detection, 4-day-old female wild-type flies were injected with FHV (20 or $200 \mathrm{TCID}_{50}$ per fly). The appearance of FHV DNA was analyzed by single-fly PCR at 6 and $12 \mathrm{~h}$ after infection and daily up to $8 \mathrm{~d}$ after infection. To determine the number of flies that generate a viral DNA form after infection with RNA virus, we infected flies with FHV or Sindbis virus or mock infected flies with $10 \mathrm{mM}$ Tris- $\mathrm{HCl}, \mathrm{pH}$ 7.5. The formation of viral DNA was monitored by single-fly PCR at $6 \mathrm{~d}$ after infection with the following pairs of primers: 69F and 1002R for FHV; and NSP1F and 913R for Sindbis virus.

For infection of flies by viral feeding, the following procedure was used for the AZT in vivo assay: $w^{1118}$ flies were fed $93 \mathrm{mM}$ AZT in $25 \%$ sucrose daily from day 2 after eclosion or were not fed AZT. At day 4, flies were fed a pure stock of FHV $\left(1 \times 10^{9} \mathrm{TCID}_{50}\right.$ per $\left.\mathrm{ml}\right)$ or Tris- $\mathrm{HCl}$ $10 \mathrm{mM}, \mathrm{pH} 7.5$ (as a control), once overnight. Survival was monitored every day for $16 \mathrm{~d}$. Flies were kept at $25^{\circ} \mathrm{C}$. At various time points, flies were collected and viral titers were calculated as described above.

For single-fly PCR, each fly was homogenized in $50 \mu$ squishing buffer (100 mM Tris- $\mathrm{HCl}$, $25 \mathrm{mM} \mathrm{NaCl}, 1 \mathrm{mM}$ EDTA, $\mathrm{pH}$ 8, and $0.2 \mathrm{mg} / \mathrm{ml}$ proteinase $\mathrm{K}$ ) and incubated for $1 \mathrm{~h}$ at $37^{\circ} \mathrm{C}$. Proteinase $\mathrm{K}$ was inactivated for $2 \mathrm{~min}$ at $95^{\circ} \mathrm{C}$. A portion of the homogenate $(1 \mu \mathrm{l})$ was treated for 30 min at $37^{\circ} \mathrm{C}$ with 10 units of DNase I (Roche) or not, followed by heat inactivation $\left(72{ }^{\circ} \mathrm{C}\right.$ for $10 \mathrm{~min})$. A portion of the sample ( $1 \mu \mathrm{l}$, corresponding to $0.1 \mu \mathrm{l}$ of the original homogenate) was analyzed by PCR.

AZT treatment. S2n or S2p cells $\left(3 \times 10^{6}\right)$ were incubated for $6-48 \mathrm{~h}$ in the presence or absence of $5 \mathrm{mM} \mathrm{AZT} \mathrm{(Sigma-Aldrich).} \mathrm{Cells} \mathrm{were} \mathrm{then} \mathrm{inoculated} \mathrm{with} \mathrm{a} \mathrm{tenfold} \mathrm{serial} \mathrm{dilution} \mathrm{of} \mathrm{FHV.} \mathrm{At}$ the time of inoculation, AZT was removed or maintained for the rest of the experiment. Viral titers were calculated by endpoint dilution as described above.

S2 cell extracts and assay of reverse-transcriptase activity. S2 cell pellets were washed once with PBS and lysed in CHAPS lysis buffer (10 mM Tris- $\mathrm{HCl}, \mathrm{pH} 7.5,400 \mathrm{mM} \mathrm{NaCl}, 1 \mathrm{mM} \mathrm{MgCl}$, 1 mM EGTA, 0.5\% CHAPS and 10\% glycerol, supplemented before use with Complete EDTA-free protease inhibitor 'cocktail' (Roche) and $1 \mathrm{mM} \mathrm{DTT}$ ). After incubation for $10 \mathrm{~min}$ at $4{ }^{\circ} \mathrm{C}$, cell 
debris were removed by centrifugation at $16,000 \mathrm{~g}$ for $10 \mathrm{~min}$ at $4{ }^{\circ} \mathrm{C}$. Supernatants were transferred to clean tubes. Total protein concentration was determined by Bradford assay (Biorad). Samples were 'snap-frozen' in liquid nitrogen and were stored at $-80{ }^{\circ} \mathrm{C}$ until use.

Reverse-transcriptase assays were carried out for $15 \mathrm{~min}$ at $25^{\circ} \mathrm{C}$ in a reaction volume of $50 \mu$ l containing $4 \mu \mathrm{g}$ S2 cell extracts or $0.05 \cup$ Superscript II (invitrogen), $320 \mathrm{ng}$ oligo(dT) (Invitrogen), $500 \mathrm{ng}$ poly(rA) (GE Healthcare) and $1 \mu \mathrm{Ci}\left[\alpha-{ }^{32} \mathrm{P}\right] \mathrm{dTTP}(3,000 \mathrm{Ci} / \mathrm{mmol}$; PerkinElmer) in $50 \mathrm{mM}$ Tris- $\mathrm{HCl}$, pH 7.5, $50 \mathrm{mM} \mathrm{KCl}, 5 \mathrm{mM} \mathrm{MgCl}$ or $0.7 \mathrm{mM} \mathrm{MnCl}_{2}, 5 \mathrm{mM}$ DTT and $0.1 \%$ Triton $\mathrm{X}-100$. Then, $5 \mu$ of each reaction was spotted in triplicate onto DE-81 paper (an ion-exchange paper that retains incorporated nucleotides but not free dNTP). Papers were washed five times with $100 \mathrm{ml}$ of $2 \times$ saline-sodium citrate solution, followed by exposure to a Phosphorlmager screen. The nucleotide analog and reverse-transcriptase inhibitor azidothymidine-triphosphate was from Bioron. Nonlinear regression and determination of halfmaximal inhibitory concentration were calculated with Prism 5 software (GraphPad).

Small-RNA and genomic libraries. Small-RNA libraries of S2 cells were constructed as described $^{44}$. For $\beta$-elimination, standard procedures were followed ${ }^{45}$. Small RNAs were treated for $10 \mathrm{~min}$ at room temperature with $25 \mathrm{mM}$ sodium periodate, followed by $\beta$-elimination for $90 \mathrm{~min}$ at $45{ }^{\circ} \mathrm{C}$ in $50 \mu \mathrm{L} 1 \times$ borax buffer ( $30 \mathrm{mM}$ borax, $30 \mathrm{mM}$ boric acid and $50 \mathrm{mM} \mathrm{NaOH}, \mathrm{pH}$ 9.5). Only small RNA with 2'-O-methyl at the extremity (a sign of Ago2 loading) that resisted the oxidation treatment were recovered by sequencing. Genomic DNA libraries of S2 cells were generated with Nextera Technology for Next-Generation Sequencing Library Preparation (Epicentre) with a fragment size centered around 400 base pairs. Libraries were sequenced (36 single reads for small RNAs and $2 \times 54$ paired-end reads for genomic DNA) on an Illumina Genome Analyzer IIx. Reads were analyzed with in-house Perl scripts.

Bioinformatics analysis of small-RNA libraries. For the detection of chimeric small RNAs, virusderived siRNA profiles were generated according a published method ${ }^{17}$. For the identification of chimeric siRNAs, small RNA reads 36 nucleotides in length were clipped for adapters with the FASTX-Toolkit suite (a collection of command line tools), with reads at least 18 nucleotides in length kept and reads in which the adaptor sequence could not be detected discarded. After removal of contaminating sequences (primers, adapters, ribosomal RNA and so on), reads in which the adaptor as clipped $\left(\sim 25 \times 10^{6}\right.$ for S2n and S2p cells, and $\sim 15 \times 10^{6}$ for S2p cells after $\beta$-elimination) were grouped by unique sequence $\left(\sim 3.6 \times 10^{5}\right.$ for S2n cells and $\sim 1 \times 10^{6}$ for S $2 p$ 
cells) with an in-house script. Each unique sequence was given a unique identifier followed by the number of sequences that it represented in the library.

First, unique sequences were filtered with Blastall software (National Center for Biotechnology Information) with the parameters '-W 9 -F F -e 1-g F', first against the Drosophila melanogaster reference genome (ftp://ftp.flybase.net/releases/FB2010 03/dmel r5.26) and then against FHV RNA1 and RNA2 (NC_004146.1 and NC_004144.1, respectively) and their defective interfering particles (GU393238.1 for RNA1, and GU393239.1, GU393240.1 and GU393241.1 for RNA2). At each filter, all the unique sequences with a high-scoring segment pair above 18 bases with 0 and 1 mismatches were eliminated. This first step eliminates all nonchimeric small RNAs with a good match in either the host genome or the virus genome.

Second, the Blastall software was used with the parameters '-e 100 -W 9 -F F -q -100 -g $F^{\prime}$ for selection, among the remaining unique sequences, those with 9-12 bases with the most similarity to FHV RNA1 or RNA2 and their defective interfering particles without gaps or mismatches. Unique sequences without similarity or with similarity of more than 12 bases were not selected. A second blast analysis was done on the selected unique sequences with the parameters '-e 10000 -W 9 -F F -q -100 -g F' for selection of the unique sequences with 9-13 bases of similarity with the Drosophila reference genome. Unique sequences without similarity or with similarity of more than 13 bases were not selected. Then, an 'in-house' script was used for comparison of the two Blastall series (versus FHV and versus Drosophila), with selection of only the unique sequence with at least 1 'hit' against FHV and 1 'hit' against Drosophila and whose positions were considered nonoverlapping. Results that allowed 0, 1 and 2 overlapping bases between the two hits were examined for all the unique sequences. Only unique sequences representing at least five reads on the original small-RNA library were retained. This second step was specifically designed to 'fish out' chimeric transcripts with high confidence.

Of note, to detect chimeric small RNAs that unambiguously map partly to Drosophila and partly to FHV, we applied very stringent mapping and filtering parameters. By doing this, we may have lost many chimeric reads that were unable to pass the filters, and thus the final numbers are low (Fig. 4d and Supplementary Fig. 6).

For comparison of small-RNA libraries with or without AZT treatment, the following procedures were used. For miRNA analysis, the mirBase database was used as reference. Mapping was done with Bowtie software, with applying a seed of $21 \mathrm{nts}$ with a maximum of 2 
mismatches. Each miRNA was quantified in the presence or absence of AZT with SAMtools. FHV small RNAs were mapped with Bowtie software for the alignment of short DNA sequences, and a maximum of two mismatches was allowed. The mapping of siRNAs was annotated for each position of FHV RNA1.

Silencing assay. S2 cells $\left(\sim 1 \times 10^{6}\right)$ were transfected with dsRNA with Effectene (QIAGEN). The dsRNA was generated by in vitro transcription from T7 promoter-flanked PCR products, with. 2 $\mu \mathrm{g}$ dsRNA used per condition in six-well plates with a $2 \mathrm{ml}$ final volume of Schneider's medium. After $3 \mathrm{~d}$ of dsRNA treatment, cells stained with propidium iodide, followed by analysis with a FACSCalibur and CellQuest Software.

RNA blot analysis. Total RNA was isolated with TRIzol (Invitrogen). RNA ( $24 \mu \mathrm{g})$ was separated by electrophoresis through $1.5 \%$ denaturing agarose gels, then transferred to a Nytran SuperCharge membrane with the Turbo Blotter system (Whatman). RNA was crosslinked to membranes by ultraviolet irradiation (Stratalinker) and was prehybridized for $2 \mathrm{~h}$ at $39{ }^{\circ} \mathrm{C}$ in ULTRAhyb-oligo buffer (Ambion). DNA oligonucleotide probes with complementary to FHV RNA1 and RNA3 and to FHV RNA2 were end-labeled with ${ }^{32} \mathrm{P}$ with $\mathrm{T} 4$ polynucleotide kinase (Fermentas), then were added to the hybridization buffer, followed by incubation overnight at $39{ }^{\circ} \mathrm{C}$. Membranes were washed several times at $39^{\circ} \mathrm{C}$ in $0.1 \times$ saline-sodium citrate with $0.1 \%$ SDS and then exposed to a Phospholmager screen. Probes were stripped by boiling of the membrane twice in $0.1 \%$ SDS for a second round hybridization with Rp49 as a 'housekeeping' control.

Quantitative real-time RT-PCR. Total RNA was extracted from S2 cells with TRIzol (invitrogen), then $1 \mu \mathrm{g}$ total RNA was treated with DNase I according to the manufacturer's instructions (Roche). The cDNA was prepared by reverse transcription with iScript Reverse Transcriptase (BioRad) with oligodT and random hexamer primers. Roche Universal Sybr Green Master Mix (Rox) and a StepOne Plus (Applied Biosystems) were used for quantitative RT-PCR. The changein-threshold values were calculated within the log-linear phase of the amplification curve with the StepOne Plus V2.2.2 software (Applied Biosystems). Quantification was normalized to that of mRNA encoding the endogenous ribosomal protein Rp49. Oligonucleotide primers were as follows:

DNA oligonucleotides ( $5^{\prime}$ to $\left.3^{\prime}\right)$ 
RNA blot

FHV1 3015-R CTTCCGGTTGTTGGAAGGC

FHV1 2970-R GCGTTCTTCGAGTGTTGGTT

FHV2 701-R CCACCGCTAGAACACCATCT

FHV2 971-R ACCATGCCTTGAGTATGGC

Rp49 465-R ACAAATGTGTATTCCGACCACG

PCR

FHV1 1-F GTTTTCGAAACAAATAAAACAGAAAAG

FHV1 27-F GCGAACCTACACAATGACTCTA

FHV1 69-F CCAGATCACCCGAACTGAAT

FHV1 1002-R CGACCGATGGAAACCAGCAGTTC

FHV1 1240-R CAGTTGGACTAATTGGTGACAC

FHV1 2537-R AACCTGCTTCATCAAATGGG

FHV1 2674-R CGCCGTCTTCATCAAACGTACA

FHV1 2970-R GCGTTCTTCGAGTGTTGGTT

FHV1 3107-R CCTCTGCCCTTTCGGGCTAGAACGGG

SIN NSP1-F AAGGATCTCCGGACCGTACT

SINV 913-R CCTTCGCAACTCACCACTGT

SIN NSP1-F TCTGCCGATCATAGCACAAG

SIN NSP2-R CTTCTTAACGCAACGCTTC

SIN NSP3-F GGATCAATTTTCGACGGAGA

SIN NSP4-R TTGAATGTCGCTGAGTCCAG

SINV 10299-F AAGGTCTTCGGAGGGGTCTA

SINV 10898-R AATGGGAATGTTCCCGTATG

DCV 724-F CCAGAGGGCGTTGTCGTCTCCCCCT 
DCV 1108-R GGGGCGATTGAACGGGTCCAGGG

DCV 3133-F GTTGCCTTATCTGCTCTG

DCV 4328-R CGCATAACCATGCTCTTCTG

DCV 4235-F CGACTCGTACTGGGGATTGT

DCV 4863-R AGGAAATCCTGGTGACGTTG

DXV-A 277-F CGTCGAGTATTAGCGGCTTC

DXV-A 767-R GCCCTACGGAGTCCACATTA

DXV-B 1493-F AGGTTGGACATCGAAACAGG

DXV-B 2175-R GGCTAGCCTCTACGGCTTTTT

DXV-B 1812-F TCAAGGCATTCGATCCCTAC

DXV-B 2330-R CCATACGCGTTGTGTATTCG

dsRNA generating PCR primers

T7 Gal80-F TAATACGACTCACTATAGGGAGAGGGCCCTTGCATGTTCACTAG

T7Gal80-R TAATACGACTCACTATAGGGAGACCTTTGAAACTGCATGACACTGG

T7 CG4572-F TAATACGACTCACTATAGGGAGACTATAGTCGCAATAAGCGGAGC

T7 CG4572-R TAATACGACTCACTATAGGGAGATATGGCATTTTGTACCTTGTGG

T7 Dcr2-F TAATACGACTCACTATAGGGAGAAAGCGGTTGTAGTTGATATCGC

T7 Dcr2-R TAATACGACTCACTATAGGGAGAAGTACGTATCCCGTAGAGCTGG

reverse transcriptase-qPCR

297-F TGGACGGACAAATTACACGA

297-R TCCGATTGGTTACCTTCCAG

blood-F GACCAAAGCCCTTGACCATA

blood-R TACTTCGCACCACGAAGTTG

micropia-F ATATTGTTCGCCCAAGTTGC

micropia-R TAATTTGCTCCGCGAAGTCT

copia-F GGAGGTTGTGCCTCCACTTA 
copia-R CTCTTGGAGACGCTTTACGG

mdg1-F AAGCCTGCCTGTTTTCAAGA

mdg1-R TGCTTCACTCTGACCCTCCT

gapdh-F TGATGAAATTAAGGCCAAGGTTCAGGA

gapdh-R TCGTTGTCGTACCAAGAGATCAGCTTC

rp49-F ATCGGTTACGGATCGAACA

rp49-R ACAATCTCCTTGCGCTTCTT

FHV1-F CCAGATCACCCGAACTGAAT

FHV1-R AGGCTGTCAAGCGGATAGAA

<jrn>42. Reed, L.J. \& Muench, H. A simple method of estimating fifty per cent endpoints.

Am. J. Hyg. 27, 493-497 (1938).</jrn>

$<$ jrn $>43$. Cherry, S. \& Perrimon, N. Entry is a rate-limiting step for viral infection in a

Drosophila melanogaster model of pathogenesis. Nat. Immunol. 5, 81-87 (2004).</jrn>

<jrn>44. Gausson, V. \& Saleh, M.C. Viral small RNA cloning and sequencing. Methods Mol.

Biol. 721, 107-122 (2011).</jrn>

$<$ jrn $>45$. Vagin, V.V. et al. A distinct small RNA pathway silences selfish genetic elements in the germline. Science 313, 320-324 (2006).</jrn> 